\title{
EDITORIALS
}

\section{Foresight is 2020: Ten Bold Predictions for the New CRISPR Year}

Rodolphe Barrangou

Whereas most people use the last few days of the year to reflect on the events that shaped the previous 12 months, I have decided to embrace the disruptiveness of CRISPR and pre-emptively offer 10 bold predictions to set the stage for what promises to be yet another eventful, perhaps pivotal, year for genome editing.

Rather than offer a linear progression of incremental insights into what is likely to come, I will channel the ability of our field to leap ahead and offer colorful, perhaps contrarian, predictions of what may (hopefully will) happen for CRISPR in the next 12 months.

1. Notwithstanding lingering technical issues (e.g., offtarget effects and immunogenicity concerns) and public apprehension, there will be several genome editing clinical successes. This year closes with promising early clinical news from Victoria Gray, the first U.S.-based sickle-cell patient treated with a CRISPR therapy. In 2020, investigational new drug filings will trend up and U.S. FDA regulators will support active recruiting for several clinical trials. I hope they will quickly generate positive results for several indications by various groups of investigators and clinicians. Both safety and efficacy for multiple indications may well be in the cards for 2020.

2. In an era of scientific skepticism, the public will embrace CRISPR and increasingly appreciate the real-world benefits of genome editing. Despite widespread misinformation, distrust in the scientific enterprise, and low confidence in scientists dedicating their lives to solving real challenges, team science will step up and share more wonderful stories. In place of sensational media headlines and overdramatized fearmongering, I am looking for the real stories of the scientists leading the revolution and of the people benefiting from it.

3. Europe catches up with the world. The good thing about science is that (sometimes) it is immune to politics_-just sound thinking, creative minds, and the scientific method. European pundits and regulators will eventually open their eyes to see the light and objectively assess the upside of CRISPR for diverse applications for their health, food, environmental, and commercial benefits. \#crispEUr

4. Rather than continue with toolbox expansion, current CRISPR tools will be put to good use. After years of next-generation Cas mining and optimization of the Cas, dCas, nCas and fused-effector domains, we will appreciate that the current tools are polished enough for most users and uses. With a useful Cas (Cas-9, 12, 13, et al.) toolbox, diverse applications (editing the genome, transcriptome, and epigenome) and optimized technologies (such as prime editing), currently available tools will be harnessed and implemented with greater urgency and less concerns about technology enhancement.

5. Beyond therapeutics. With increasingly promising signs of clinical success, the potential of genome editing will be unleashed for livestock, crops, and even trees for a more sustainable agriculture and healthier planet. Organisms spanning most branches of the tree of life will be enhanced for broad societal benefits.

6. Cooler heads prevail. Despite the continuation of intellectual property disputes and interference proceedings, progress toward commercialization of actual products and the need to split large pies that cannot be eaten whole will compel dominant parties to partner and split the proceeds. After all, science is a team sport hinging on scientific collaborations, and the community spirit will behoove key players and leaders to play nice.

7. Business dealmakers join the fray. Investors and strategists will be intrigued by deflated stock prices given the extraordinary potential. Underperforming CRISPR stocks and underwhelming financial performancesymptoms that have affected the biotech sector as a whole-have not dampened the upside of CRISPR. The recent report of sickle-cell clinical data pushed valuations significantly higher. It would not be a surprise to see pharma competitively bid for early-stage CRISPR companies and undervalued tickers. Will we see our first CRISPR start-up acquisition in 2020? \#CRISPRM\&A 
8. CRISPR responsibility. Two major reports on germline editing, from the National Academies/Royal Society and the World Health Organization, will be released in 2020. We hope the reports will coordinate, with all the voices of CRISPR being heard, so we can build consensual and broadly acceptable frameworks to ensure we use CRISPR responsibly, especially regarding usage in human embryos for germline editing. The public has asked for it, and the community has been working on it. The science versus society gap will be bridged.

9. CRISPR fatigue. Despite all the fanfare, I suspect there will be some CRISPR fatigue in 2020: after years of Addgene-fueled democratization, Odin-fed biohacking, and inexorable publication and citation growth, the rate at which CRISPR is expanding will start to slow down and plateau. This is not necessarily a bad thing, as most users in need have already adopted this technology.

10. CRISPR goes global. Beyond academic scientists blazing new trails and investors hunting for new technologies, nations will define visions and strategies to expand and build national CRISPR portfolios to harness the bio-economy and keep up with the competition. Leading nations have claimed a stake in the scientific literature and intellectual property arenas, but as business appetite broadens and commercialization success advances, it will be perilous not to seize editing opportunities.

So get some rest to prepare for an exciting and eventful year ahead!

\section{Partnering with bioRxiv}

Rodolphe Barrangou

When we launched The CRISPR Journal in 2018, we warmly welcomed the submission of preprints posted on servers such as bioRxiv. It has taken a while, but we are pleased to announce that The CRISPR Journal has joined bioRxiv's B2J (bioRxiv-to-journal) "one-click" program. From now on, authors who post their preprint to bioRxiv will be able to transfer the preprint directly into The CRISPR Journal's manuscript submission system. Why is this important?

Following several unsuccessful attempts to launch preprint servers in the life sciences over the past two decades, the movement has rapidly gained in popularity in recent years. A tipping point came in 2015, when visionary cell biologist Ron Vale published a commentary arguing for the need to accelerate the dissemination of research data in biology in an era of increasing complexity and delays in the top peer-review journals. ${ }^{1}$ Vale followed that up by convening an important workshop at the Howard Hughes Medical Institute, which catalyzed the benefits and momentum of supporting preprints, and a second commentary (with Anthony Hyman) advocating for the benefits of separating dissemination (one of the advantages of preprints) from validation (the central purpose of peer-review journals). ${ }^{2}$

Not every researcher in the life sciences is convinced; the surge of preprints posted to bioRxiv, a nonprofit repository hosted by the Cold Spring Harbor Laboratory, is approach- ing 3,000 per month. However, the monthly total is still a fraction of the papers posted to arXiv, the physics preprint repository. The brief history of CRISPR is riven with several examples of important manuscripts that were delayed during peer review (see Gilles Vergnaud's "My CRISPR Story" in this issue for a glaring example). It is tempting to wonder how the history of the field might be seen if researchers posted their preprints first and worried about journal selection and peer review later. I personally see much value to the classical peer review process in terms of improving manuscripts and their narratives and granting journals the privilege to manage the dissemination and announcement of novel and highly impactful results. But I also note the merits of the timeliness and transparency afforded by preprints.

We hope the added convenience of our new arrangement with bioRxiv will encourage more researchers among the growing number of CRISPR scientists to give The CRISPR Journal the chance to handle peer review and publication of their work. It couldn't be easier-it's just one click away! Consider it our holiday gift to you.

\section{References}

1. Vale RD. Accelerating scientific publication in biology. Proc Natl Acad Sci 2015;112:13439-13446. DOI: 10.1073/pnas.1511912112.

2. Vale RD, Hyman AA. Point of view: priority of discovery in the life sciences. elife 2016;5:e16931. DOI: 10.7554/eLife.16931. 